Case Report

\title{
Odontogenic Cyst with Verrucous Proliferation Exhibiting Melanin Pigmentation
}

\author{
Nidhi Manaktala, ${ }^{1}$ Karen Boaz, ${ }^{1}$ Krupa Mehta Soni, ${ }^{2}$ Srikant Natarajan, ${ }^{1}$ Junaid Ahmed, \\ Keshava Bhat, ${ }^{4}$ Nandita Kottieth Pallam, ${ }^{1}$ and Amitha Juanita Lewis ${ }^{1}$ \\ ${ }^{1}$ Department of Oral Pathology, Manipal College of Dental Sciences, Manipal University, Mangalore, Karnataka 575001, India \\ ${ }^{2}$ University of Colorado School of Dental Medicine, Aurora, CO, USA \\ ${ }^{3}$ Department of Oral Medicine and Radiology, Manipal College of Dental Sciences, Manipal University, Mangalore, \\ Karnataka 575001, India \\ ${ }^{4}$ Department of Oral and Maxillofacial Surgery, Srinivas Institute of Dental Sciences, Mangalore, Karnataka 575001, India
}

Correspondence should be addressed to Karen Boaz; karen.boaz@manipal.edu

Received 3 November 2016; Revised 25 January 2017; Accepted 9 February 2017; Published 20 March 2017

Academic Editor: Piero Tosi

Copyright (C) 2017 Nidhi Manaktala et al. This is an open access article distributed under the Creative Commons Attribution License, which permits unrestricted use, distribution, and reproduction in any medium, provided the original work is properly cited.

\begin{abstract}
Verrucous proliferation arising from odontogenic cysts is a rare entity. We report an unusual case of an infected odontogenic cyst with verrucous proliferation and melanin pigmentation in a 13-year-old male patient who presented with an intraoral swelling in relation to impacted teeth 26 and 27. The enucleated lesion was diagnosed as an odontogenic keratocyst and the patient died within two years of presentation due to multiple recurrences. The clinical, radiological, and microscopic features of the lesion are presented with an attempt to discuss the etiopathogenesis. The case hereby reported is uncommon with only eight cases reported in the literature.
\end{abstract}

\section{Introduction}

Odontogenic cysts have a diverse morphology. Though their origin and clinical presentations have been well documented, they continue to puzzle the pathologists with few rare clinicopathological presentations. Verrucous proliferation in a cystic lining is one such rarity. To the best of our knowledge only eight cases of odontogenic cysts with verrucous proliferation have been reported in English literature [18], of which six were reported as verrucous carcinomas arising in odontogenic cysts. We describe a case of an infected odontogenic cyst exhibiting verrucous proliferation and melanin pigmentation. It is also the first case of mortality reported among the several cases reviewed till date.

\section{Case Report}

A 13-year-old male patient reported with a complaint of swelling and fetid discharge in upper left posterior tooth region since few months. On clinical examination, a solitary swelling of the left maxillary molar segment with a nonulcerated mucosa (except for a draining sinus) was evident with missing 26 and 27. The swelling was firm, had well-defined borders, and was nontender on palpation. Radiographically, a well-defined radiolucent cystic lesion surrounding impacted 26 and 27 was seen (Figure 1). Based on the age and the clinical findings, a provisional diagnosis of infected dentigerous cyst was made. The impacted teeth were surgically extracted and the cystic lining was enucleated and submitted for histopathological examination.

\section{Gross and Histopathological Findings}

Grossly, the excised tissue measured about $6.6 \times 2.5 \times 2.2 \mathrm{~cm}$ and the whitish-brown cystic lining showed focal areas of nodular/papillary proliferation (Figure 2).

Microscopic examination of the specimen revealed a keratin-filled cystic lumen lined by a discontinuous five to fifteen cells thick para- to orthokeratinised epithelium (Figures 3 and 4) with keratin plugging and whorls showing 


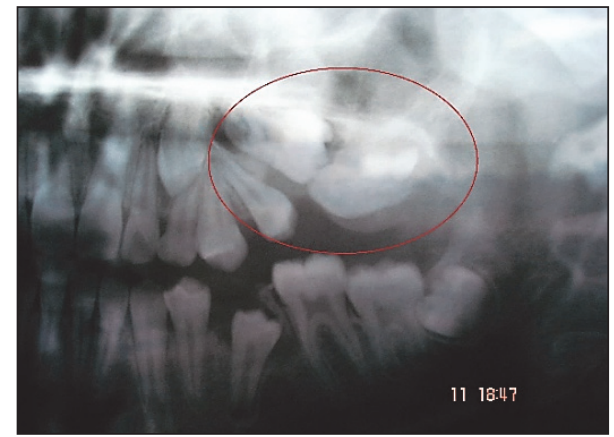

FIGURE 1: Cystic lesion (encircled) is seen surrounding the impacted 26, 27 and developing 28.

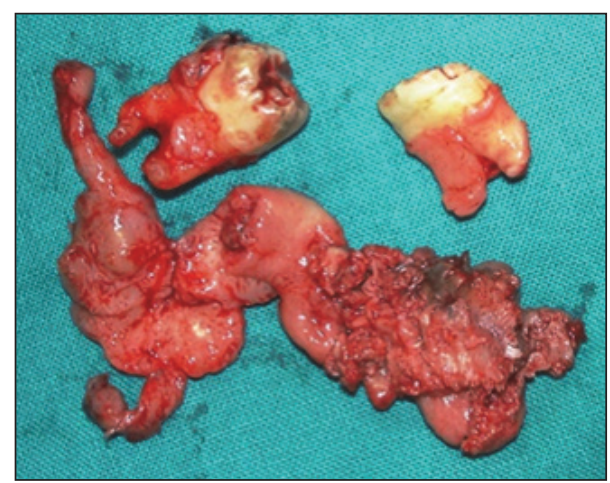

FIGURE 2: Excised specimen showing proliferative cystic lining and extracted 26 and 27.

(inset). The cystic epithelium showed areas of hyperplasia and broad bulbous rete ridges (Figure 4) along with areas of hypergranulosis. Koilocytic changes were also seen along with increased melanin pigmentation and melanin incontinence (inset). There was no evidence of fungal hyphae in the tissue sample as assessed by periodic acid Schiff's stain.

The supporting collagenous connective tissue capsule showed moderate to dense mixed inflammatory cell infiltrate and numerous dilated blood vessels. Many melanophages were also seen juxta-epithelially (Figure 4). Focal areas of leukocytic exocytosis were seen in the overlying epithelium.

The intraosseous location of the cyst in close association with impacted teeth suggested an odontogenic origin. The histopathological appearance was closest in compatibility to the diagnosis of an odontogenic keratocyst while not being typical of it. After due diligence and recognising the variation from classical appearances, a diagnosis of inflamed odontogenic cyst exhibiting verrucous proliferation was given. Despite the presence of a prominent verrucous component, a distinct lack of endophytic growth and "pushing" epithelial ridges precluded a diagnosis of verrucous carcinoma. Gel electrophoresis (GeneRuler ThermoFisher Scientific) confirmed the presence of human papilloma virus DNA in the tissue specimen. The lesion reportedly recurred thrice over the next two years and curettage was performed but the specimen was unavailable to us for histopathological examination at each of these recurrences. The lesion subsequently extended

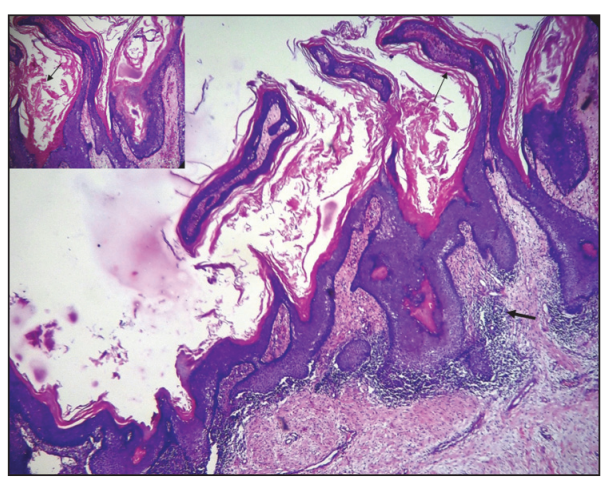

FIGURE 3: Cystic lumen lined by para- to orthokeratinised stratified squamous epithelium projecting into the lumen in the form of finger-like projections and filled with keratin. Capsule exhibiting dense inflammation (arrow) (4x, H\&E).

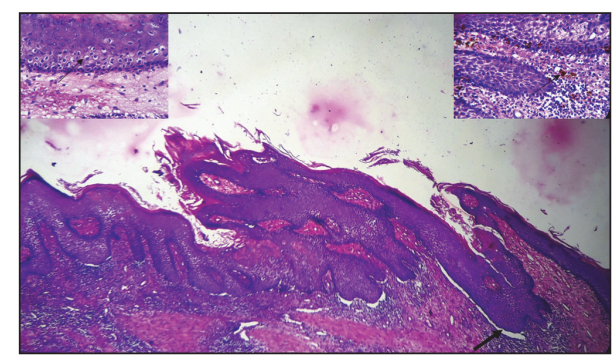

FIGURE 4: Photomicrograph showing parakeratinised stratified squamous epithelium with bulbous rete ridges lining the cystic lumen. Spinous and superficial cells exhibiting koilocytic changes (left inset); basal and suprabasal cells showing melanin pigmentation (right inset) (10x, H \& E).

to the nasoethmoid region involving the orbit and perforating the base of skull anteriorly. The lesion eventually metastasized to the brain resulting in death.

\section{Discussion}

Verrucal proliferation in odontogenic lesions is an extremely rare occurrence with only a handful of cases reported in the literature till date. The demographic, clinical, radiographic, and histopathological diagnostic information of the eight reported cases of odontogenic cysts with verrucous proliferation (including verrucous carcinoma) has been compared in Table 1. These (including the present case) have been seen amongst a wide age group, ranging from 13 to 74 years. They show male preponderance $(4: 1)$ and are more common in the maxilla as compared to the mandible. Anterior maxilla has been reported to be affected more commonly [1-8].

Aldred et al. (2002) reported the first case of verrucous proliferation in an odontogenic cyst that occurred in a 13year-old female patient in right maxillary alveolus, in relation to an impacted canine and the root of the lateral incisor. It was enucleated and did not recur [3]. The second case was reported by Ueeck et al. (2007) in the left mandibular ramus of a 46-year-old male with evidence of residual 


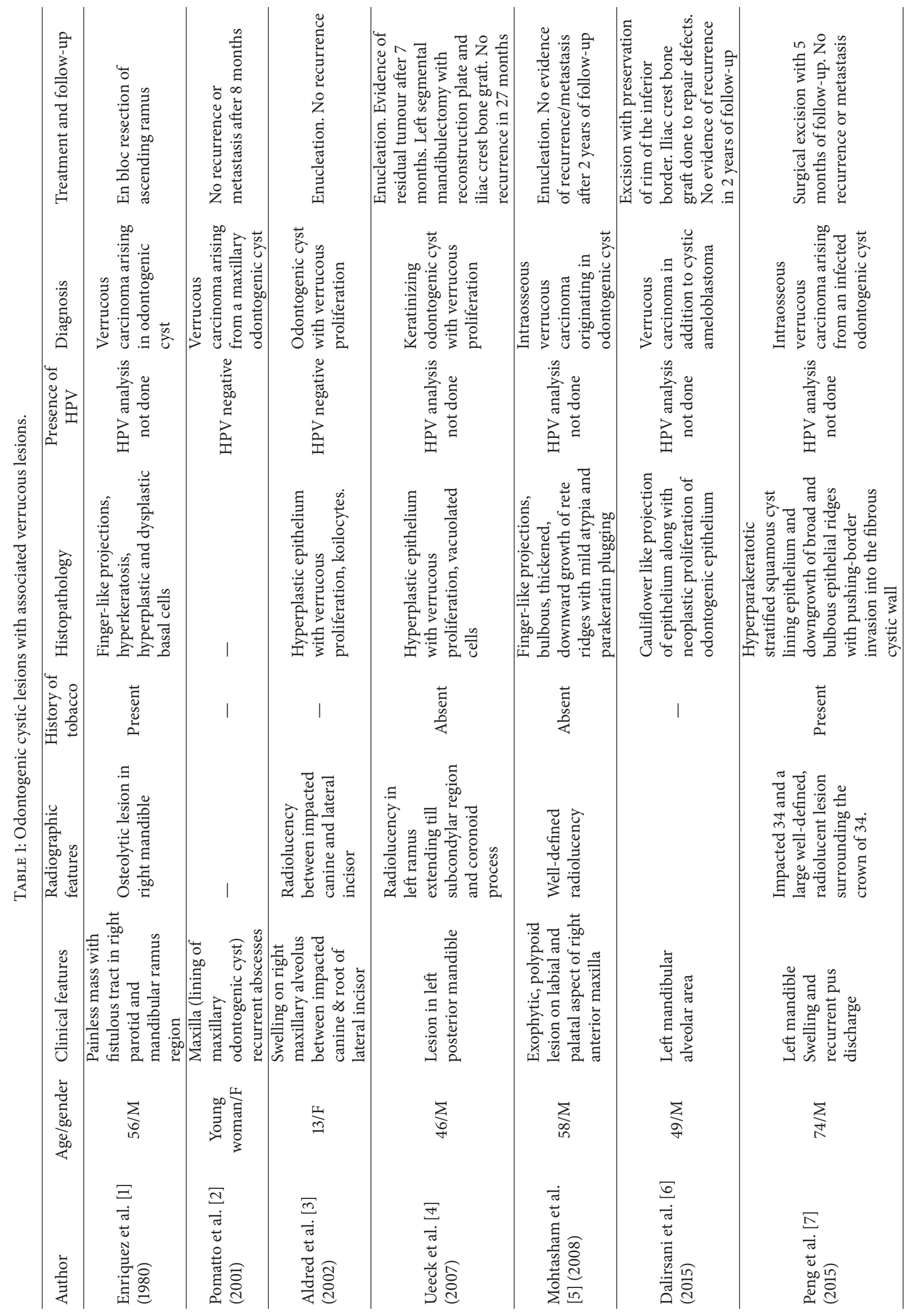




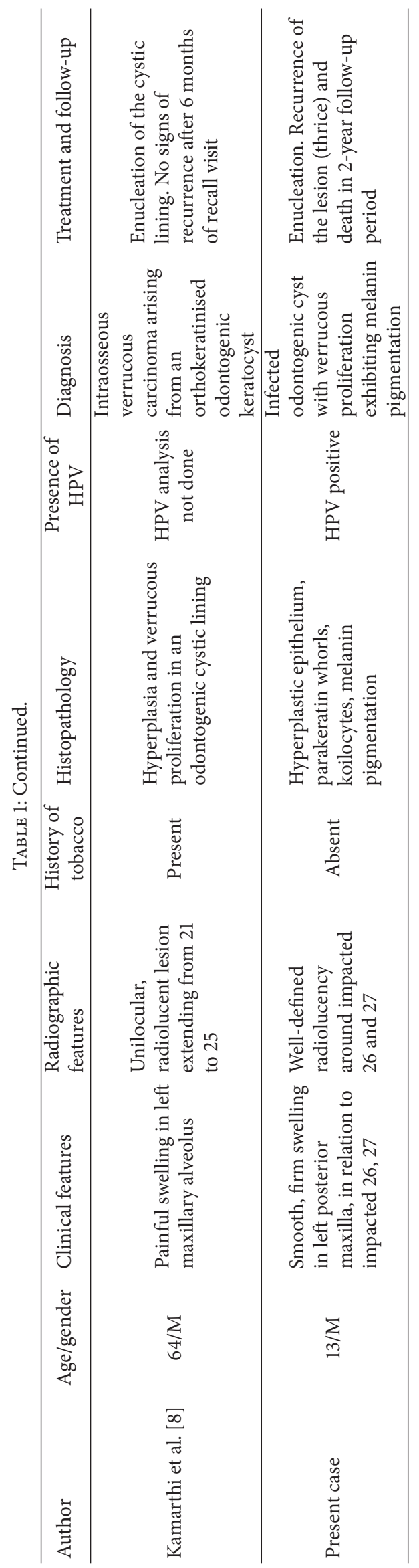


lesion 7 months after enucleation. Subsequent left segmental mandibulectomy and reconstruction were performed with no recurrence even after follow-up of 27 months [4]. The present case was an aggressive one as there were multiple recurrences of the lesion ultimately leading to the death of the patient.

Conventionally, a cystic lesion like an OKC/KCOT is known for its aggression but is not commonly known to metastasize. Cases of OKC have been described in the literature wherein maxillary lesions have extended to involve the orbit, skull base, and infratemporal fossa [9-11].

The occurrence of verrucous proliferation in an intraosseous cyst causes one to speculate on the supposed cause. Various etiologies for verrucous growth in odontogenic lesions proposed thus far include the presence of HPV (human papilloma virus), candidal infection, and the habit of tobacco consumption as these factors have correlated well with oral mucosal verrucous lesions [4]. However, projection of the same pathogenesis for an intraosseous odontogenic cyst is not tenable. Although tobacco plays a role in oral mucosal verrucal lesions, it is less likely to be a cause of verrucous proliferation in an intraosseous odontogenic cyst $[5,6]$. Additional studies are needed to elucidate whether tobacco carcinogens in the bloodstream may circulate to chronically inflamed sites to induce verrucal proliferation [7]. While it has been suggested that candida produces nitrosamines which serve as neoplastic stimuli in verrucous lesions as stated by Ueeck et al. [4], the present case showed absence of candidal organisms in the tissue. It is therefore doubtful that candida species can be implicated in the presentation of verrucal growth in an intraosseous cyst.

Human papilloma virus (HPV) is strongly associated with oral verrucous lesions, verrucous cysts seen in skin, and even verrucous proliferation in ameloblastoma [12-14]. HPV produces the early gene proteins E6 and E7, which affect the keratinocyte cell cycle. E6 binds to the keratinocyte protein p53 (a regulating protein that inhibits keratinocyte cell division). Once p53 is bound to E6, it is degraded, resulting in an uninhibited keratinocyte mitosis and epithelial proliferation. The E7 protein binds the retinoblastoma protein $(\mathrm{Rb})$ and in a similar manner disturbs the keratinocyte cell division cycle [15]. In the present case, HPV could have induced verrucous proliferation in the cystic lining as histologically there was focal koilocytic change as well as presence of the viral genome (detected in the tissue using gel electrophoresis). We speculate that the probable path for entry of the HPV in an intraosseous lesion (preexistent?) may have been via the thin lining of the maxillary sinus as the relatively thicker oral epithelium may have offered more resistance to such an invasion of the virus. Alternatively, the presence of the draining sinus might have offered a path for entry of HPV entry resulting in the verrucous proliferation. The distinctive histology of a verrucous carcinoma, namely, the endophytic growth and pushing epithelial rete ridges, was conspicuously missing. In cases of verrucous cysts of dermis, Soyer et al. agreed with the hypothesis proposed by Rous and Beard that stated that keratinocytes infected by Shope papillomavirus (SPV) can descend into the dermis and induce verrucous cysts [13]. In addition to this, Ueeck et al. emphasized the views by Kahn who had suggested that HPV in intrabony lesions may be acquired in utero or at parturition and involve the invaginating primary enamel organ [4].

Another histopathologically notable variation seen in the present case was melanin pigmentation and melanin incontinence which is unusual in an odontogenic lesion. One may surmise that the origin of the dental lamina (and further, that of the odontogenic cyst) from primitive oral mucosa may justify the presence of melanocytes $[16,17]$. Neural crest cells play an important role in odontogenesis as reflected in the reciprocal induction that occurs between inner enamel epithelium and cells of the dental papilla (which originate from ectomesenchyme, a derivative of neural crest cells). Differentiation of melanocytes in this neural crest cell-rich zone is thus possible. Lawson et al. have also stated that mucosal melanocytes tend to accumulate near the attachment of dental lamina to the oral epithelium and suggested the dual role of neural crest cells in primary induction (melanocyte differentiation) as well as in the formation of tooth anlage [18]. Additionally, as most of the cases have been reported in Asian and Black patients, it is possible that the increased pigmentation may be attributed to racial variation [16].

Aldred et al. have suggested the use of the term "verrucous odontogenic cyst" [3]. However, the name "keratinising odontogenic cyst with verrucous proliferation" suggested by Ueeck et al. [4] is preferable even though it tends to veil the ambiguity of whether the lining of the odontogenic cyst undergoes verrucous proliferation or it is a "verrucous odontogenic cyst arising de novo," which could be a new entity.

Regardless of whether such a lesion arose de novo or through transformation of a preexisting lesion, an "odontogenic cyst with verrucous proliferation" is certainly a rare entity. Of the nine cases reported with verrucous growth (including the present case) two have been reported in 13year-old children and six were reportedly signed out as verrucous carcinoma. The patient in the present case succumbed within two years of diagnosis of the lesion due to recurrences, thus giving an insight into the aggressive behaviour of the lesion which was most likely to be due to infection by HPV. While only three of the nine cases (including present case) were assessed for presence of HPV, positivity for the HPV genome was seen only in our case (see Table 1).

The report of the present case emphasizes the need for careful enucleation and thorough histopathological examination of aggressive cystic lesions. It also warrants the need for running advanced diagnostics to identify presence of HPV infection as it might be a factor determining the prognosis of such lesions.

\section{Conclusion}

Odontogenic cysts are known to present with numerous variations, verrucous proliferation, and pigmentation being one of the rarest histological presentations. These lesions tend to be aggressive in behaviour even bearing a diagnosis of verrucous carcinoma.

The likely association of these lesions with HPV infection warrants careful and thorough investigations including PCR for detection of HPV genome and PET scans as follow-up for 
recurrence. Through the present report we record the ninth case of odontogenic cyst with verrucous proliferation, with a review of the previous cases in terms of histopathology and possible etiopathogenesis.

\section{Conflicts of Interest}

The authors declare that they have no conflicts of interest.

\section{References}

[1] R. E. Enriquez, B. Ciola, and S. L. Bahn, "Verrucous carcinoma arising in an odontogenic cyst. Report of a case," Oral Surgery, Oral Medicine, Oral Pathology, vol. 49, no. 2, pp. 151-156, 1980.

[2] E. Pomatto, V. Carbone, D. Giangrandi, and V. Falco, "Primary intraosseous verrucous carcinoma developing from a maxillary odontogenic cyst: case report," Tumori, vol. 87, no. 6, pp. 444446, 2001.

[3] M. J. Aldred, A. A. Talacko, P. G. Allan, and M. Shear, "Odontogenic cyst with verrucous proliferation," Journal of Oral Pathology and Medicine, vol. 31, no. 8, pp. 500-503, 2002.

[4] B. A. Ueeck, B. M. Woo, J. C. B. Stewart, and L. A. Assael, "Keratinizing odontogenic cyst with verrucous proliferation," Journal of Oral and Maxillofacial Surgery, vol. 65, no. 3, pp. 585588, 2007.

[5] N. Mohtasham, F. Babazadeh, and H. Jafarzadeh, "Intraosseous verrucous carcinoma originating from an odontogenic cyst: a case report," Journal of Oral Science, vol. 50, no. 1, pp. 91-94, 2008.

[6] Z. Dalirsani, F. Falaki, N. Mohtasham, and L. V. Mostaan, "Oral verrucous carcinoma and ameloblastoma: a rare coincidence," Iranian Journal of Otorhinolaryngology, vol. 27, no. 79, pp. 159163, 2015.

[7] C.-Y. Peng, Y.-F. Huang, M.-Y. Lu, Y.-H. Lee, and C.-H. Yu, "Intraosseous verrucous carcinoma arising from an infected dentigerous cyst-a case report," Journal of the Formosan Medical Association, vol. 114, no. 8, pp. 764-768, 2015.

[8] N. Kamarthi, S. Palakshappa, V. Wadhwan, and R. Mohan, "Intraosseous verrucous carcinoma arising from an orthokeratinized odontogenic keratocyst: a report of a rarest entity," Contemporary Clinical Dentistry, vol. 7, no. 3, pp. 416-419, 2016.

[9] I. T. Jackson, Z. Potparic, M. Fasching, W. I. Schievink, K. Tidstrom, and K. Hussain, "Penetration of the skull base by dissecting keratocyst," Journal of Cranio-Maxillofacial Surgery, vol. 21, no. 8, pp. 319-325, 1993.

[10] R. Chuong, R. B. Donoff, and W. Guralnick, "The odontogenic keratocyst," Journal of Oral and Maxillofacial Surgery, vol. 40, no. 12, pp. 797-802, 1982.

[11] S. F. Worrall, "Recurrent odontogenic keratocyst within the temporalis muscle," British Journal of Oral and Maxillofacial Surgery, vol. 30, no. 1, pp. 59-62, 1992.

[12] J. A. Regezi, J. J. Sciubba, and R. C. K. Jordan, Verrucal-Papillary Lesions, Elsevier, 5th edition, 2009.

[13] H. P. Soyer, D. Schadendorf, L. Cerroni, and H. Kerl, "Verrucous cysts: histopathologic characterization and molecular detection of human papillomavirus specific DNA," Journal of Cutaneous Pathology, vol. 20, no. 5, pp. 411-417, 1993.

[14] W. F. P. Van Heerden, E. J. Van Rensburg, E. J. Raubenheimer, and E. H. Venter, "Detection of human papillomavirus DNA in an ameloblastoma using the in situ hybridization technique,"
Journal of Oral Pathology and Medicine, vol. 22, no. 3, pp. 109$112,1993$.

[15] V. Kumar, A. Abbas, and N. Fausto, "Neoplasia," in Robbins and Cotran. Pathologic Basis of Disease, pp. 269-290, Elsevier, New York, NY, USA, 7th edition, 2005.

[16] S. S. Farahani and M. Lotfalian, "A pigmented dentigerous cyst in a patient with multiple dentigerous cysts of the jaws: a case report," Journal of Contemporary Dental Practice, vol. 8, no. 5, pp. 85-91, 2007.

[17] A. Warter, G. George-Diolombi, M. Chazal, and A. Ango, "Melanin in a dentigerous cyst and associated adenomatoid odontogenic tumor," Cancer, vol. 66, no. 4, pp. 786-788, 1990.

[18] W. Lawson, I. F. Abaci, and F. G. Zak, "Studies on melanocytes. V. The presence of melanocytes in the human dental primordium: an explanation for pigmented lesions of the jaws," Oral Surgery, Oral Medicine, Oral Pathology, vol. 42, no. 3, pp. 375-380, 1976. 


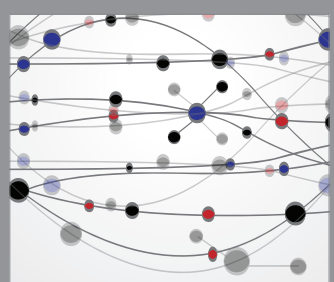

The Scientific World Journal
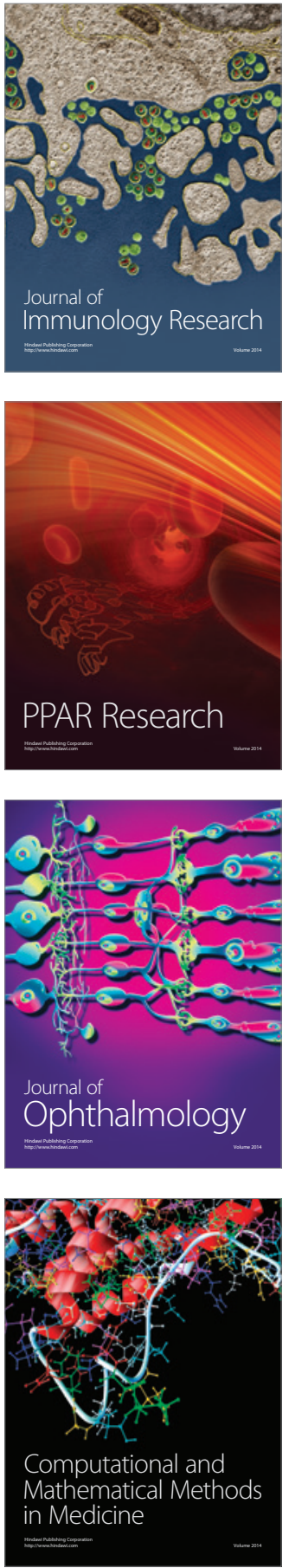

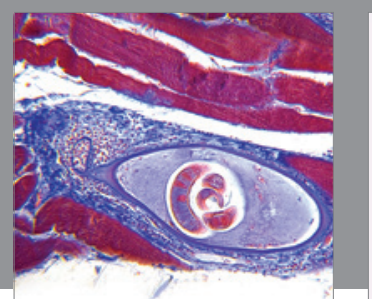

Gastroenterology Research and Practice
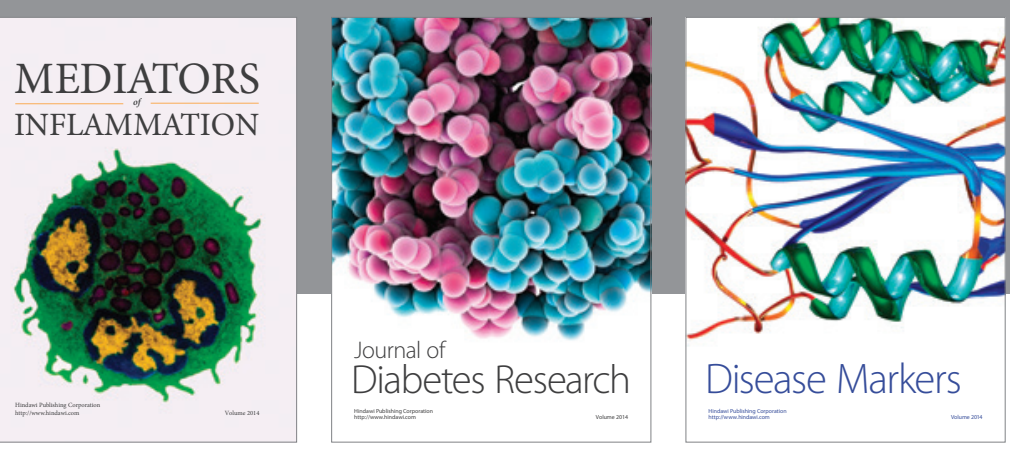

Disease Markers

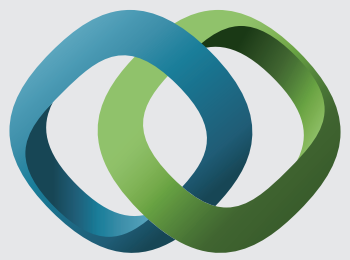

\section{Hindawi}

Submit your manuscripts at

https://www.hindawi.com
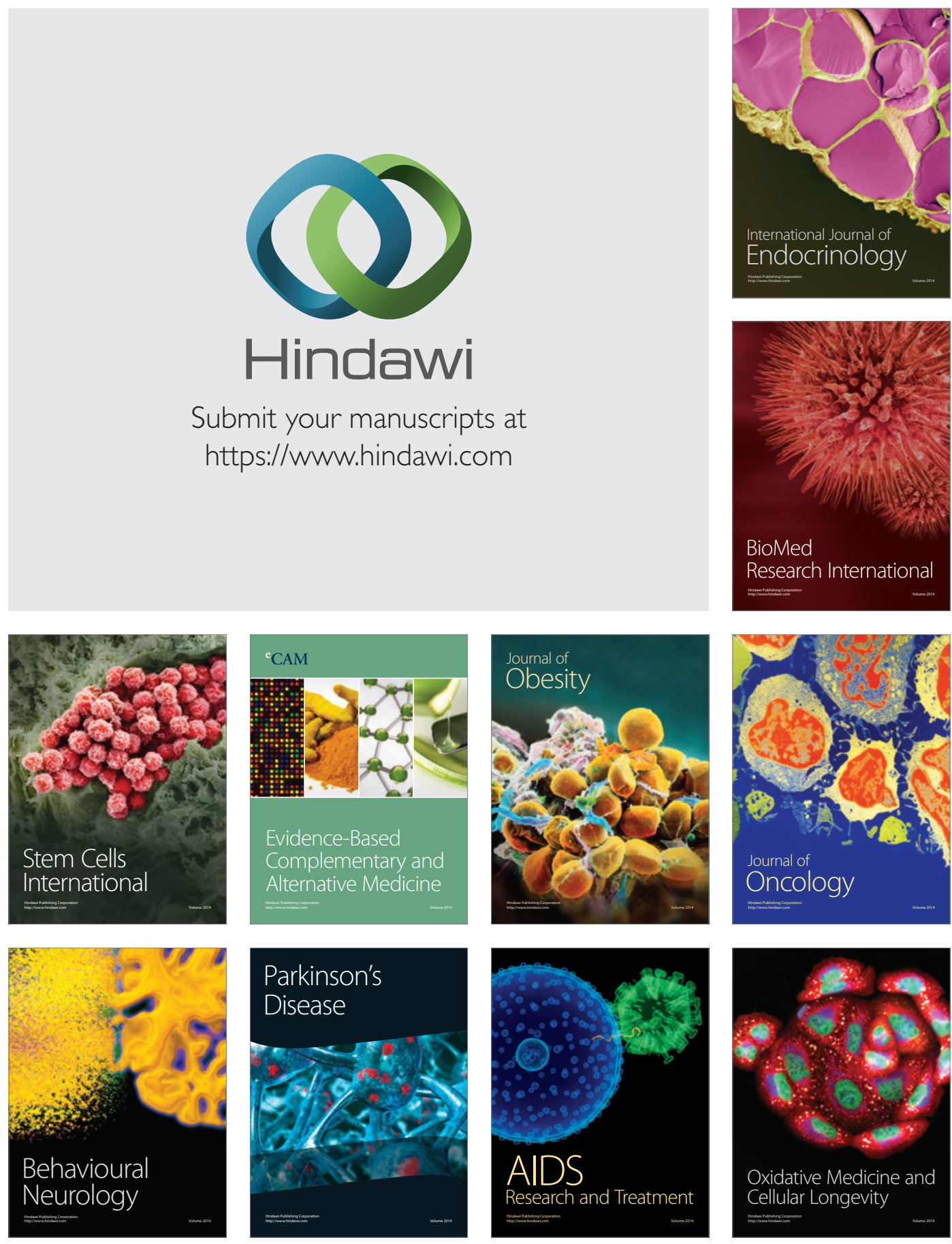